\title{
The influence of color on the taste perception of carbonated water preparations
}

\author{
ARNOLD HYMAN \\ University of New Haven, West Haven, Connecticut 06516
}

\begin{abstract}
Tasteless food dyes were used to impart three different colors-red, yellow, and brown-to carbonated water and white (colorless) birch beer. Subjects were requested to taste the preparations and judge the flavor in order to assess the influence of the colors on taste perception. This influence was clearly in evidence when the subjects tasted the birch beer, but little effect was seen with the carbonated water.
\end{abstract}

The color of food has been shown to influence taste perception. A short review may be found in Pangborn (1967). Experiments have been of two types: those requiring subjects to make differential judgments along a single dimension (Pangborn, 1960; Pangborn, Berg, \& Hansen, 1963; Romeu \& DeVincente, 1968), and those requiring subjects to identify the taste of the stimulus presented (Hall, 1958). Since the present experiment is of the latter type, the Hall study is of particular interest.

Hall (1958) employed sherbets of different flavors, each presented in three colors: the commonly associated color, an inappropriate color, and white or uncolored. The subjects were generally successful in identifying flavors when they were associated with their "normal" colors but made many identification errors when the color was lacking or inappropriate. This study, however, failed to include a control for possible taste effects of the food colors and used very cold stimuli. The latter factor could have been a problem, since Bartoshuk and Hooper (Note 1) have reported that taste sensitivity for sucrose is reduced by cold temperatures. The present study, therefore, required subjects to identify taste stimuli of "unusual" colors presented at room temperature, and it also included a control condition to determine if the food colors imparted a taste to the stimuli.

\section{GENERAL METHOD}

\section{Subjects}

The subjects were 100 human adults, mostly of college age. Known smokers and foreign-born people were excluded. ${ }^{1}$

\section{Materials}

Materials included commercial carbonated water, white (colorless) birch beer, manufactured by the Cott Corporation, and Durkee brand food colors, manufactured by the SCM Corporation.

The technical assistance of Louis DiManto and Maxine Tusa is acknowledged. Financial assistance was provided by the University of New Haven. Valerie Moore is thanked for secretarial assistance, and the critical advice of Tom Mentzer and Mike York is gratefully acknowledged.

\begin{tabular}{l} 
Subject \\
Quality of Soda: \\
Good \\
\cline { 2 - 7 }
\end{tabular}

What the soda tastes like:

Figure 1.

\section{Test Procedure}

Each subject tasted only one sample. The subjects were told that we were testing soda for a local company, ${ }^{2}$ and they were instructed to taste the sample of soda provided and to fill out a questionnaire (Figure 1) regarding the type and palatability of the soda. Thirty cubic centimeters of soda were provided in a small plastic cup at room temperature, and the subject was told to drink as much as he wished. In the blindfolded groups, subjects were fitted with an adjustable blindfold with eye pads before they were presented with the soda. Pilot work indicated that the blindfold was effective.

\section{Preparation of Samples}

Color was imparted to the carbonated water and birch beer by opening the bottles, adding appropriate amounts of the food colors, and resealing the bottles. Labels were removed. Red, yellow, and brown sodas were prepared. The red resembled cherry soda in appearance, the brown, birch beer or root beer, and the yellow, lemon soda (particularly, a brand called "MelloYellow"). Directions for imparting the colors are given in the appendix.

\section{EXPERIMENT 1}

\section{Method}

Experiment 1 involved the tasting of carbonated water. The following conditions were employed: blindfolded tasting of uncolored carbonated water, blindfolded tasting of brown carbonated water, nonblindfolded tasting of red carbonated water, and nonblindfolded tasting of brown carbonated water. Twenty subjects were employed in each of the first two conditions, and 10 each in the last two.

\section{Results}

Table 1 shows the results of Experiment 1. The uncolored carbonated water was identified by all but 
Table 1

Judgments of Subjects Tasting Carbonated Water

\begin{tabular}{|c|c|c|c|c|c|c|c|}
\hline \multicolumn{4}{|c|}{ Blindfolded } & \multicolumn{4}{|c|}{ Nonblindfolded } \\
\hline \multicolumn{2}{|l|}{ Clear } & \multicolumn{2}{|l|}{ Brown } & \multicolumn{2}{|l|}{ Red } & \multicolumn{2}{|l|}{ Brown } \\
\hline Response & $\mathrm{N}$ & Response & $\mathrm{N}$ & Response & $\mathbf{N}$ & Response & $\mathrm{N}$ \\
\hline Club Soda or Seltzer & 17 & Club Soda or Seltzer & 15 & Seltzer or Club Soda & 7 & Seltzer or Club Soda & 7 \\
\hline Flat 7-Up & 1 & Tonic Water & 2 & Weak Cherry Soda & 1 & Cola & 1 \\
\hline \multirow{3}{*}{ Tonic Water } & 2 & Diet Coke & 1 & Cough Medicine & 1 & Flat Root Beer & 1 \\
\hline & & Diet Orange & 1 & "Close-Up" Toothpaste & 1 & Flat Cream Soda & 1 \\
\hline & & Diet Soda & 1 & & & & \\
\hline
\end{tabular}

three of the blindfolded subjects. Fifteen of the 20 blindfolded subjects who tasted the brown preparation also identified the stimulus correctly.

Regarding the nonblindfolded judgments, the effect of visual input due to the color of the stimulus may be seen in six of the subjects, but it is a weak and even questionable effect on two accounts: Not many subjects were affected, and most of those that were gave "weak" responses (e.g., "flat root beer," "weak cherry soda"). Palatability ratings were poor for all colors.

\section{Discussion}

The food colors are advertised as having no taste, but nevertheless, we felt obliged to put this claim to an experimental test by having subjects taste brown seltzer blindfolded. The brown preparation was chosen because all three of the food dyes used in the study are components of the brown color (see appendix). Thus, using the brown preparation was felt to be the most convenient and least effortful way to run this type of control.

The results indicate clearly that the food coloring did not impart any taste to the carbonated water. Although 5 of the 20 subjects who tasted the brown preparation did assign an actual taste to it (a response that we shall call a false positive), this by no means indicates that the food colors imparted a taste, since the clear carbonated water also produced three false positives when tasted by blindfolded subjects. The difference between the two is obviously not significant. Furthermore, it is likely that the types of instructions used could have contributed to the occurrence of the false positives. The occurrence of false positives in many different kinds of experiments has, of course, been thoroughly documented.

\section{EXPERIMENT 2}

\section{Method}

Experiment 2 involved the tasting of white birch beer. Sight was permitted except in the first condition. Twenty subjects were used in each condition, and the standard testing procedure was used. The following conditions were employed: blindfolded tasting of uncolored birch beer, tasting of uncolored birch beer, tasting of red birch beer, tasting of yellow birch beer, and tasting of brown birch beer.

\section{Results}

In the analysis of the data from Experiment 2, "root beer," "birch beer," and "sarsaparilla" were considered equivalent responses. Consequently, "root beer" and "sarsaparilla" were considered correct identifications.

Table 2

Judgments of Subjects Tasting Uncolored Birch Beer

\begin{tabular}{lrrrrr}
\hline \multicolumn{1}{c}{ Blindfolded } & & & \multicolumn{2}{c}{ Nonblindfolded } \\
\cline { 1 - 1 } \cline { 5 - 6 } \cline { 5 - 6 } Response & Nirch (or Root) Beer & & Response & N \\
Dr. Pepper & 12 & & Birch (or Root) Beer & 14 \\
Group of Sodas Mixed & 2 & & Pepto-Bismol & 2 \\
Strong Flavor, No Real Quality & 1 & & Mint Flavored & 1 \\
Liquid Medicine and Cold Duck & 1 & & Lemon and Lime & 1 \\
Coke & 1 & No Particular Flavor & 1 \\
Chewing Gum & 1 & & & 1 \\
Lemony-Like Bland 7-Up & 1 & & \\
\hline
\end{tabular}

Table 3

Judgments of Subjects Tasting Colored Birch Beer

\begin{tabular}{|c|c|c|c|c|c|}
\hline \multicolumn{2}{|l|}{ Red } & \multicolumn{2}{|l|}{ Yellow } & \multicolumn{2}{|l|}{ Brown } \\
\hline Response & $\mathbf{N}$ & Response & $\mathbf{N}$ & Response & $\mathbf{N}$ \\
\hline Birch (or Root) Beer* & 5 & Birch (or Root) Beer & 10 & Birch (or Root) Beer & 10 \\
\hline Birch (Root) Beer or Cherry Soda & 2 & Listerine & 3 & Cream Soda & 7 \\
\hline Carbonated Cherry Cough Medicine & 2 & Wintergreen and 7-Up & 1 & Cream, Dr. Pepper, and Sprite & 1 \\
\hline Cherry Soda & $\overline{1}$ & Wintergreen/Birch Beer & 1 & Spearmint Life Savers & 1 \\
\hline Dr. Pepper & 1 & Wintergreen & 1 & Root Beer or Cough Syrup & 1 \\
\hline Mint Flavor & 2 & Pepto-Bismol & 1 & & \\
\hline Dentist Office Substance Used to Clean Teeth & $\overline{1}$ & Cough Medicine & 1 & & \\
\hline Mouthwash From Dentist & 1 & Licorice & 1 & & \\
\hline Watery and Weak Cough Syrup & 1 & & & & \\
\hline Seltzered Pepto-Bismol & 1 & & & & \\
\hline Crest Toothpaste & 1 & & & & \\
\hline Teaberry Gum & 1 & & & & \\
\hline Medicine & 1 & & & & \\
\hline
\end{tabular}

*Includes two "sarsaparilla" responses. 
Table 4

Summary of Palatability Judgments of Experiment 2

\begin{tabular}{lcccccc}
\hline & \multicolumn{5}{c}{ Rank } & Mean \\
\cline { 2 - 5 } & 1 & 2 & 3 & 4 & 5 & Rank \\
\hline Red & 1 & 3 & 9 & 6 & 1 & 3.15 \\
Brown & 2 & 6 & 5 & 5 & 2 & 2.95 \\
Yellow & 5 & 3 & 6 & 4 & 2 & 2.75 \\
Uncolored & 4 & 6 & 6 & 3 & 1 & 2.55 \\
Blindfolded & 2 & 6 & 7 & 4 & 1 & 2.80 \\
\hline
\end{tabular}

Tables 2 and 3 show the results of Experiment 2. It is clear that the taste perception of a majority of the subjects was affected by the visual input. The red color was the strongest, with 13 subjects failing to identify the birch beer and 2 being unable to decide whether it was birch beer or cherry soda. Ten subjects in the yellow group and 10 in the brown group also failed to identify the birch beer, as well as 8 subjects in the blindfolded group and 6 in the uncolored group. If we classify the two uncertain responses in the red group as incorrect, since these subjects were obviously influenced by the red color, then a chi-square analysis shows that the red group had significantly more incorrect identifications than the blindfolded group $\left(\chi^{2}=5.01\right.$, $\mathrm{p}<.05)$, as well as the uncolored group $\left(\chi^{2}=8.12\right.$, $\mathrm{p}<.01))^{3}$ The eight "cream soda" responses in the brown group were also significant, since only one other such response occurred in the entire experiment. Similarly, the three "Listerine" responses in the yellow group are notable.

Table 4 shows the palatability ratings for all the groups. The median test revealed no significant difference in the ratings.

\section{Discussion}

The large number of "cream soda" responses, eight in all, to the brown stimulus was unexpected. Brown is one of the "usual" colors of birch beer or root beer, and it was expected to yield mostly correct identifications. Presumably, the "cream soda" responses competed with the correct response. However, although cream soda is usually colored brown, it is flavored with vanilla, and such a flavor does not seem to be related to birch beer or root beer. Further investigation is indicated regarding the relationship between the two flavors. In the final analysis, the subjects tasting the brown stimulus, as a group, did no better than the blindfolded subjects. The visual input, which should have been an aid in identification, created a competing response, "cream soda," that canceled out whatever advantage might have accrued as a result of the additional clue. This conclusion is supported by the fact that the subjects who tasted the uncolored birch beer with vision made more correct identifications than the brown group. (Although this difference is not statistically significant, we feel there is a strong trend in that direction.) More important, the identification errors of the uncolored group and the blindfolded group were random, whereas those of the brown group tended to be directional, "toward" cream soda.

The greater ability of the red color than the yellow to generate color-oriented responses is not understood. It is noteworthy that the yellow color produced no lemon responses, a factor that could account for the difference. We may hypothesize that cherry flavor bears greater resemblance to birch beer than does lemon flavor. The yellow preparation did, however, generate three clearly color-oriented "Listerine" responses, since the color of the yellow preparation closely resembled Listerine.

As can be seen, many responses in Experiment 2 were attempts to describe the taste of the stimulus as resembling something other than soda. Typical examples are: "mouthwash from dentist," "licorice," "Listerine," "spearmint Lifesavers," and "Pepto-Bismol" (see Table 3). A reasonable hypothesis is that these subjects were confused by the lack of agreement between taste and color, thought the soda had a strange taste, and did their best to describe that taste in the most effective terms they could think of. A future modification of this experiment would restrict responses to soda flavors only. This would require the subjects to give more precise judgments.

Although effects of visual input have been clearly demonstrated, the failure of a large number of subjects to be influenced by color should be discussed. Although the discussion is speculative, it is instructive. We may assume that one parameter of a subject's response would be the amount of previous contact he had had with birch beer or similar soda. Extensive experience would presumably increase the subject's ability to detect the birch beer, whereas lack of experience would have the opposite effect. It is also likely that the subjects' general taste sensitivity varied. Finally, we may assume that subjects differ in the general extent to which visual inputs influence taste perception.

\section{GENERAL DISCUSSION}

The results of the present experiment corroborate the findings of the previous experiments, demonstrating the influence of color on taste perception (Hall, 1958; Pangborn, 1960; Pangborn et al., 1963; Romeu \& DeVicente, 1968), but they provide a better test of the effect of color on taste perception for two basic reasons.

The first reason is based upon the general difference between one-dimension studies, and studies requiring a taste identification. A comparison between the present study and that of Romeu and DeVicente (1968) is useful at this point. In the study of Romeu and DeVicente, subjects tasted orange soda that differed in color in the yellow-orange range, and they were asked to make a discrimination along a sweet-sour dimension. On the other hand, subjects in the present experiment were shown three widely different colors and were permitted to give an unrestricted answer. Further, we have demonstrated a stronger effect of the visual stimulus than Romeu and DeVicente did; subjects in the present study were moved by the color stimuli to completely misjudge the flavor of the substance being tasted (calling the birch beer such things as "cherry soda" or "cream soda"). In the Romeu and DeVicente study, the subjects had no such opportunity, since they were asked only to find subtle differences in the taste of what they were told was orange soda.

The second reason for the superiority of the present experiment is based on the use of the blindfolded control groups. Although the Hall (1958) study required identification of the taste stimuli, it did not include a test to determine if the food colors themselves imparted a taste, as did the present experiment. In addition, Hall did not require subjects to taste the unadulterated stimulus blindfolded in order to obtain a baseline level of incorrect identifications.

The stronger effects in the present experiment were generated with birch beer as the taste stimulus, but the use of carbonated water produced very weak, or even questionable, effects. Apparently, carbonated water has too little taste of any sort to permit an interaction between the taste stimulus and the visual stimulus. For this reason, no yellow color was employed with carbonated water and only 10 subjects were assigned to each of the other two groups; it was felt that no additional information would have been generated with additional experimentation. 
Limitations of the present experiment involve the lack of control over the taste stimulus. We cannot specify the ingredients that gave the soda its flavor, nor can the taste intensity be quantified in any way. Although these limitations do not negate the basic findnigs of the present study, we hope to overcome them in future studies by controlling the chemical composition of the taste stimuli.

\section{REFERENCE NOTE}

1. Bartoshuk, L. M., \& Hooper, J. E. Effects of temperature on the tastes of $\mathrm{NaCl}$, citric acid, quinine, sucrose, and saccharin. Paper presented at the annual meeting of the Society for Neuroscience, Los Angeles, October 1981.

\section{REFERENCES}

Hall, R. L. Flavor study approaches at McCormick and Co., Inc. In A. D. Little (Ed.), Flavor research and food acceptance. New York: Reinhold, 1958.

Panaborn, R. M. Influence of color on the discrimination of sweetness. American Journal of Psychology, 1960, 73, 229-238.

Pangborn, R. M. Some aspects of chemo-reception in human nutrition. In M. R. Kare \& O. Maller (Eds.), The chemical senses and nutrition. Baltimore, Md: Johns Hopkins Press, 1967.

Pangborn, R. M., Bera, H. W., \& Hansen, B. The influence of color on discrimination of sweetness in a dry table wine. American Journal of Psychology, 1963, 76, 492-495.

Romev, J. V., \& DeVicente, M. L. S. Influyen los colores en el sabor? Revista Interamericana de Psicologia, 1968, 2, 143-147.

\section{NOTES}

1. Smokers were excluded because smoking is known to affect taste. Foreign born were excluded because of the likelihood that their taste experiences were very different from native Americans.

2. This minor deception was adopted in order to lend the experiment a certain amount of verisimilitude.

3. Statistical theory frowns upon the use of a single sample for more than one statistical test, and some authorities contend that Experiment 2 should be analyzed with a 5 by 2 chi square. However, this test yields a nonsignificant result $\left(\chi^{2}=3.24\right)$, the acceptance of which, in the opinion of the author, would constitute a Type II error: The nonsignificant statistical results for the yellow and brown groups "dilute" the chi square and prevent the detection of the significant effect in the red group.

Appendix

Formulas for Coloring the Soda

\begin{tabular}{lc}
\multicolumn{2}{c}{ Formulas for Coloring the Soda } \\
\hline Red & $2.0 \mathrm{cc}$ Red Coloring \\
Yellow & $2.0 \mathrm{cc}$ Yellow Coloring \\
& $.4 \mathrm{cc}$ Green Coloring \\
Brown & $.4 \mathrm{cc}$ Yellow Coloring \\
& $.7 \mathrm{cc}$ Red Coloring \\
\hline
\end{tabular}

Note-All colors were added to 1 liter of birch beer or seltzer.

(Received for publication February 15, 1983.) 